\title{
Photoinduced directional domain sliding motion in peptide hydrogels promotes ectodermal differentiation of embryonic stem cells
}

\author{
Zhifei Cheng ${ }^{1 \dagger}$, Shanshan $\mathrm{Nai}^{2 \dagger}$, Shuxin Song ${ }^{1}$, Lingyi $\mathrm{Chen}^{2^{*}}$ and Zhilin $\mathrm{Yu}^{{ }^{1 *}}$
}

\begin{abstract}
Mechanical cues present in the stem cell niche resulting from intracellular processes or external force sources significantly affect the basic functions of stem cells such as self-renewal and differentiation. Creation of artificial cellular matrices exhibiting intrinsic mechanical cues generated by mechanical movements remains scarce. Herein, we reported on mechanically dynamic hydrogel matrices undergoing photo-induced directional domain sliding movement and their role in regulating embryonic stem cell (ESC) differentiation. The mechanically dynamic hydrogels were prepared via the self-assembly of an alternating hydrophilic and hydrophobic peptide with a photocaged cysteine residue. Upon light irradiation, the assemblies of the caged peptide were converted to non-equilibrated non-caged peptide bilayers that underwent the directional domain sliding motion induced by the thermodynamically favorable hydrophobic collapse transition. Culturing murine ESCs on the mechanically dynamic hydrogels resulted in biased differentiation toward the ectodermal lineage. We further showed that the mechanically dynamic hydrogels stimulated the translocation of a mechanotransduction protein Yes-associated protein (YAP) into the nucleus, implicating a potential mechanotransduction mechanism for the biased differentiation of ESCs. The finding of the biased ectodermal differentiation of ESCs induced by the mechanically dynamic hydrogels implies the great potency of the mechanically dynamic hydrogels as biomaterials for disease therapy and tissue regeneration in the future.
\end{abstract}

Keywords: hydrogels, peptide self-assembly, mechanical movement, stem cell differentiation, photo-response

\section{INTRODUCTION}

Mechanical cues present within cell local microenvironment regulate cell behavior and fate due to their critical role in cell communication [1-3]. In particular, mechanical signals in the stem cell niche resulting from intracellular processes or external force sources significantly affect the basic functions of stem cells, such as selfrenewal and differentiation [4-8]. This fact stimulates the development of biocompatible hydrogels exhibiting extrinsic or intrinsic mechanical signals as biomimetic extracellular matrices (ECM) for stem cell-based organogenesis, thus leading to great potency in tissue engineering and regeneration [9-13]. Alternative to externally applied physical forces, the intimate relationship between the elasticity of matrices and their capability in transferring force to cells indicates that controlling the intrinsic stiffness of artificial matrices allows for tuning the mechanical signals applied to stem cells and thereby guiding their differentiation [14-18]. This interpretation inspires creation of a considerable number of hydrogels with tunable mechanical properties, i.e., stiffness, for controlling and manipulating stem cell fate [19-24]. Despite the progress achieved over the past decade in hydrogels with tunable stiffness, creation of hydrogel matrices exhibiting mechanical cues resulting from intrinsic mechanical movements remains challenging, and the effect of underlying movements within hydrogels on stem cell fate is still ambiguous. Herein, we reported on mechanically dynamic peptide hydrogels undergoing photoinduced directional domain sliding movement and

\footnotetext{
${ }^{1}$ Key Laboratory of Functional Polymer Materials, Ministry of Education, State Key Laboratory of Medicinal Chemical Biology, Institute of Polymer Chemistry, College of Chemistry, Nankai University, Tianjin 300071, China

${ }^{2}$ State Key Laboratory of Medicinal Chemical Biology, Tianjin Key Laboratory of Protein Sciences, National Demonstration Center for Experimental Biology Education and College of Life Sciences, Nankai University, Tianjin 300071, China

${ }^{\dagger}$ These authors contributed equally to this work.

* Corresponding authors (emails: lingyichen@nankai.edu.cn (Chen L); yzh026@nankai.edu.cn (Yu Z))
} 
investigated the differentiation of embryonic stem cells (ESCs) guided by the mechanical movement within hydrogels.

Mechanical movement is an intrinsic behavior for many biomacromolecules and sustains their functions in distinct fundamental physiological processes such as DNA replication and ATP synthesis. These biological mechanical movements have inspired creation of artificial systems undergoing stimulus-responsive motions and exhibiting great potency in nanomedicine including controlled drug release, membrane transport, and disease detection [25-29]. In particular, artificial mechanical movements can generate large strains or stress with a high force density compared to biological motors [30]. Therefore, anchoring mechanical movement systems on the surface also allows for reversible bending and stretching of the microscopic microcantilever beams in mechanical actuators or the unidirectional transportation of macroscopic liquid drops via controlling the surface energy [31,32]. These examples demonstrate that incorporation of mechanical movements into hydrogel matrices potentially allows for force generation and thereby affecting the stem cell fate due to the resulting mechanical signals. Inspired by the sliding motion between myosin and actin proteins during muscle contraction and extension, which has been recognized as the most representative biological mechanical movement for force generation, we incorporated the directional domain sliding motion into peptide assemblies to create the mechanically dynamic hydrogels.
For this purpose, we designed and synthesized an alternating hydrophilic and hydrophobic amphiphilic peptide (VEC-CNB) containing a cysteine residue caged with a photo-cleavable $\alpha$-carboxy-2-nitrobenzyl (CNB) protection group (Scheme 1). It is worth noting that the interactions of peptides can be precisely tailored via rational design of peptide sequences to create well-defined nanostructures [33]. Based on the previous reports [34], the noncovalent interactions between alternating hydrophilic and hydrophobic domains consisting of eight residues are able to promote their self-assembly. Therefore, we hypothesized that during the self-assembly of peptide VEC-CNB, the hydrophilicity of the CNB-caged cysteine residue allows it to serve as the stopper for the hydrophobic collapse involving 8-residue-length segments [35], leading to two flexibly protruded segments within the formed bilayer structures. However, peptide VEC forms the bilayers stabilized by the hydrophobic interactions involving the full-length sequence due to the relative hydrophobicity of cysteine residue. Hence, exposing the bilayer assemblies of peptide VEC-CNB to light irradiation cleaves the photocage groups, thereby generating non-equilibrated VEC bilayers that undergo the directional domain sliding motion promoted by the thermodynamically favorable hydrophobic collapse transition. As a result, hydrogelation of the self-assembly of peptide VEC-CNB and photo-cleavage of the cage groups lead to formation of the mechanically dynamic hydrogel VECMD undergoing mechanical movement (Scheme 1), which might exhibit several advantages serving as artifi-

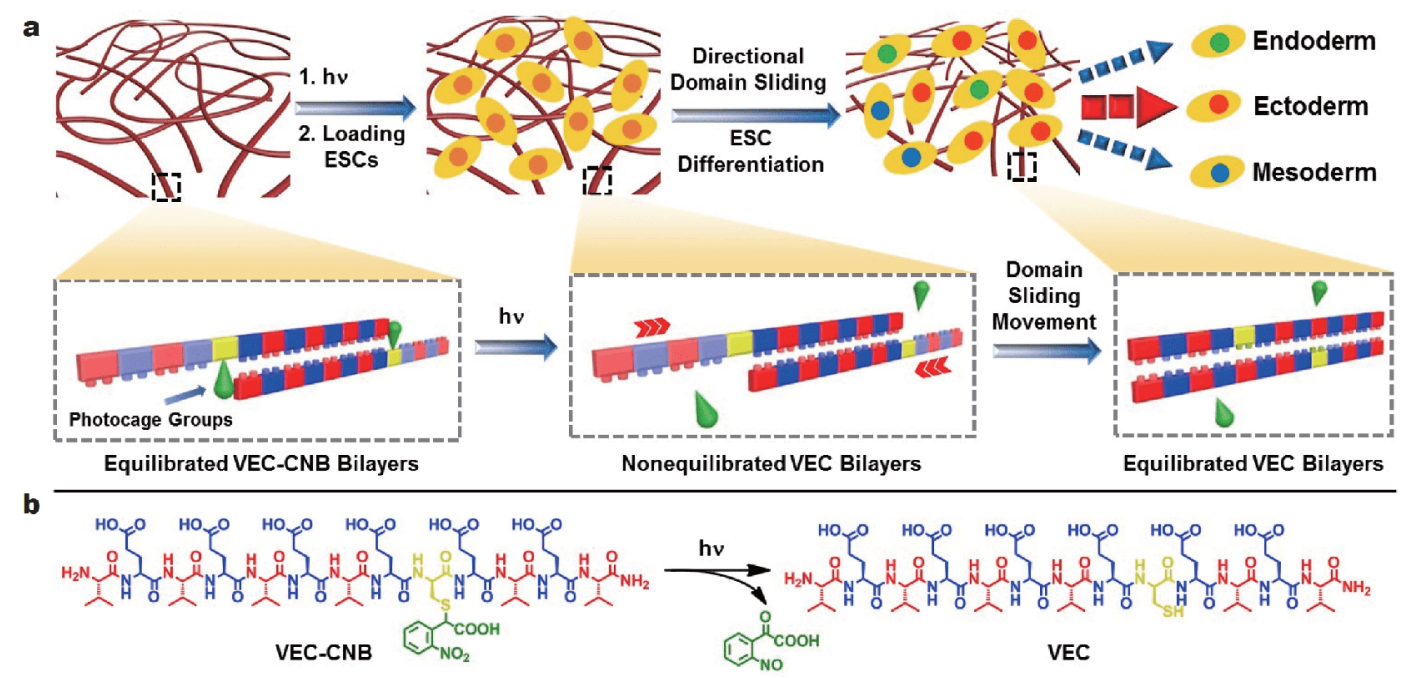

Scheme 1 Photoinduced mechanical dynamic hydrogels. (a) Schematic representation of creation of mechanically dynamic (MD) hydrogels undergoing photo-induced directional domain sliding motion and their utilization as artificial matrices for promoting the biased differentiation of ESCs into ectodermal cells associated with the directional mechanical motion; (b) chemical structures of peptide VEC-CNB containing a CNB photocage group and its photo-cleavage into peptide VEC. 
cial ECM. While the peptide component renders the great cytocompatibility of hydrogels utilized as biomaterials, the self-assembly of alternating polar and nonpolar peptides into nanofibril networks conventionally gives rise to hydrogels with tunable mechanical properties associated with peptide sequences [36]. In addition, utilization of light as stimulus to promote mechanical movement within hydrogels reserves the spatial and temporal resolution for precise control over the distribution of mechanical movements within the hydrogels [37-39], which results in the implications of the mechanically dynamic hydrogels as smart materials in the future. Based on these considerations, we employed the mechanically dynamic hydrogel VEC-MD as artificial ECM and investigated the effect of the directional mechanical movements within hydrogels on the differentiation of murine ESCs. At the same time, two thermodynamically static hydrogels prepared from equilibrated assemblies of peptides VEC-CNB and VEC also served as cellular matrices to elucidate the effect of mechanical movements on cell fate.

\section{EXPERIMENTAL SECTION}

\section{Peptide synthesis}

The starting material peptide VEC was synthesized via standard Fmoc solid-phase peptide synthesis (SPPS) using the CEM Liberty Blue peptide synthesizer, accompanied with the catalyst of $N, N^{\prime}$-diissopropylcarbodiimide (DIC). Piperidine (20\%) in N,N-dimethylformamide (DMF) solution was used to carry out the Fmoc deprotection during SPPS. Peptide on resin was cleaved from resin by using a mixture of trifluoroacetic acid (TFA)/ thioanisole/ethanedithiol/anisole in a ratio of 90:5:3:2 for $3 \mathrm{~h}$. After cleavage, the filtered solution was concentrated by rotary evaporation, and the crude peptide was precipitated from cold diethyl ether $\left(-20^{\circ} \mathrm{C}\right)$. The crude peptide was then purified by preparative reverse phase high performance liquid chromatography (RP-HPLC) equipped with a Durashell C18 column $(\mathrm{C} 18,10 \mu \mathrm{m}$, $150 \AA$, $30 \times 150 \mathrm{~mm})$. A gradient from “ $5 \%$ acetonitrile + $95 \%$ water" to "95\% acetonitrile $+5 \%$ water" was used as the eluent at a flow rate of $10 \mathrm{~mL} \mathrm{~min}{ }^{-1}$. The purified peptide VEC was converted to peptide VEC-CNB by reacting with 2-bromo-2-(2-nitrophenyl)acetic acid (BNPA), which was prepared by a modified protocol reported previously [35]. Under a nitrogen atmosphere, an aqueous solution $(10 \mathrm{~mL})$ of mixed BNPA (91.2 mg, $0.35 \mathrm{mmol}$ ) and bis-tris-propane (BTP) (566 mg, $2 \mathrm{mmol})$ was added to the aqueous solution $(10 \mathrm{~mL})$ of VEC (148 mg, $0.1 \mathrm{mmol}$ ) in a sealed Schlenk flask via syringe. Following adjusting $\mathrm{pH}$ of the reaction solution to 8.5 via aqueous ammonium hydroxide, the reactant was stirred overnight at room temperature in dark. Upon completion of the reaction, solvent was removed by lyophilization. Dispersing the resulting solid in $\mathrm{CHCl}_{2}$ $(50 \mathrm{~mL})$ by sonication and collecting the precipitate by centrifugation allowed to remove the impurities arising from BNPA and BTP reagents. The dispersion-centrifugation process was repeated again. The collected precipitate was dissolved in basic aqueous solution. Adjusting the solution $\mathrm{pH}$ to 4 by aqueous $\mathrm{HCl}$ led to precipitation and collecting the precipitate by centrifuge gave rise to the crude product VEC-CNB. The crude peptide was then purified by preparative reverse phase HPLC utilizing the identical condition for the purification of peptide VEC.

\section{Stock solutions of peptides}

All the samples for the conformational and assembling characterization of peptides VEC-CNB and VEC were prepared from the stock solutions. In the case of peptide VEC-CNB, we initially dissolved the lyophilized powder of peptide VEC-CNB in basic water $(\mathrm{pH}$ 10.0) mediated by aqueous $\mathrm{NH}_{4} \mathrm{OH}$. After adjusting the solution $\mathrm{pH}$ to 5.6 by diluted $\mathrm{HCl}$ solution, the resulting solution was heated at $80^{\circ} \mathrm{C}$ for $30 \mathrm{~min}$ and cooled down to room temperature automatically, leading to the stock solution of peptide VEC-CNB with a concentration of $2 \mathrm{mmol} \mathrm{L}^{-1}$. In the case of peptide VEC, upon UV light $(\lambda=365 \mathrm{~nm})$ irradiation at room temperature for $3 \mathrm{~h}$, peptide VEC$\mathrm{CNB}$ in the annealed solution was quantitatively converted to peptide VEC. The resulting solution of peptide VEC was annealed, yielding the stock solution of peptide VEC with a concentration of $2 \mathrm{mmol} \mathrm{L}^{-1}$.

\section{Circular dichroism (CD) spectroscopy}

$\mathrm{CD}$ experiments were performed on a spectrometer (Biologic MOS-500) using $0.2 \mathrm{~cm}$ quartz cuvettes at $25^{\circ} \mathrm{C}$. All scans were recorded with a wavelength interval of $1.0 \mathrm{~nm}$ and an acquisition time of $1 \mathrm{~s}$ in the wavelength range of 190-250 nm. CD samples of peptides VEC-CNB and VEC at a concentration of $100 \mu \mathrm{mol} \mathrm{L}^{-1}$ were prepared by diluting the stock peptide solutions $\left(2 \mathrm{mmol} \mathrm{L}^{-1}\right)$.

\section{Fourier transform infrared (FTIR) spectroscopy}

FTIR experiments were carried out using a Tensor II FTIR spectrometer (Brucker). All FTIR samples of peptides VEC-CNB and VEC were prepared by dropping the stock solution of peptides $\left(2 \mathrm{mmol} \mathrm{L}^{-1}\right)$ on the quartz 
detector under the light source. The FTIR signals from the wavenumber region of 4000 to $400 \mathrm{~cm}^{-1}$ were collected at room temperature, and only the region corresponding to the vibration absorption of amide I was shown in Fig. 1c.

\section{Transmission electron microscopy (TEM)}

TEM images were taken by a Tecnai G2 F20 microscope with an accelerating voltage of $100 \mathrm{kV}$. TEM samples of peptides VEC-CNB and VEC were prepared by diluting the stock solution of peptides to $100 \mu \mathrm{mol} \mathrm{L}^{-1} .10 \mu \mathrm{L}$ of the diluted peptide solution was pipetted onto the surface of a carbon-coated copper grid for $5 \mathrm{~min}$ and blotted by filter paper. Subsequently, $10 \mu \mathrm{L}$ of $2 \mathrm{wt} \%$ uranyl acetate was placed on the grid for staining and blotted by filter paper after $2 \mathrm{~min}$. The grid was dried in a desiccator prior to measurement.

\section{Atom force microscopy (AFM)}

AFM studies were carried out on a Bruker ICON instrument under the tapping mode. AFM samples of peptides VEC-CNB and VEC were prepared by diluting the stock solution of peptides to a concentration of $100 \mu \mathrm{mol} \mathrm{L}^{-1}$ and depositing $(10 \mu \mathrm{L})$ on the freshly cleaved mica surfaces for $5 \mathrm{~min}$. The retained solution on mica was removed by filter paper and the samples were dried in a desiccator prior to AFM experiments.

\section{Hydrogel VEC-CNB}

A solution of peptide VEC-CNB $\left(12 \mathrm{mmol} \mathrm{L}^{-1}\right)$ was prepared by dissolving the lyophilized powder of peptide VEC-CNB in ammonia water ( $\mathrm{pH} 10)$ and adjusting the $\mathrm{pH}$ to 5.6 by aqueous $\mathrm{HCl}$. The resulting solution was annealed for thermodynamic equilibration of the assemblies of peptide VEC-CNB. 10 equiv. of $\mathrm{CaCl}_{2}$ was added to the annealed solution of peptide VEC-CNB $\left(12 \mathrm{mmol} \mathrm{L}^{-1}\right)$, leading to the thermodynamically static hydrogel VEC-CNB.

\section{Intermediate hydrogel VEC}

To prepare hydrogels VEC and VEC-MD, an intermediate hydrogel VEC was prepared by adding 6 equiv. of $\mathrm{CaCl}_{2}$ into the annealed solution of peptide VEC-CNB $\left(12 \mathrm{mmol} \mathrm{L}^{-1}\right)$ and irradiating with UV light $(365 \mathrm{~nm})$ for $6 \mathrm{~h}$ to quantitatively cleave the cage groups.

\section{Hydrogels VEC and VEC-MD}

Annealing the intermediate hydrogel VEC yielded a viscous solution of peptide VEC. Addition of an additional 4 equiv. of $\mathrm{CaCl}_{2}$ into the viscous solution led to the thermodynamically static hydrogel VEC. Mechanically dynamic hydrogel VEC-MD was prepared by adding an additional 4 equiv. of $\mathrm{CaCl}_{2}$ solutions to the intermediate hydrogel VEC.
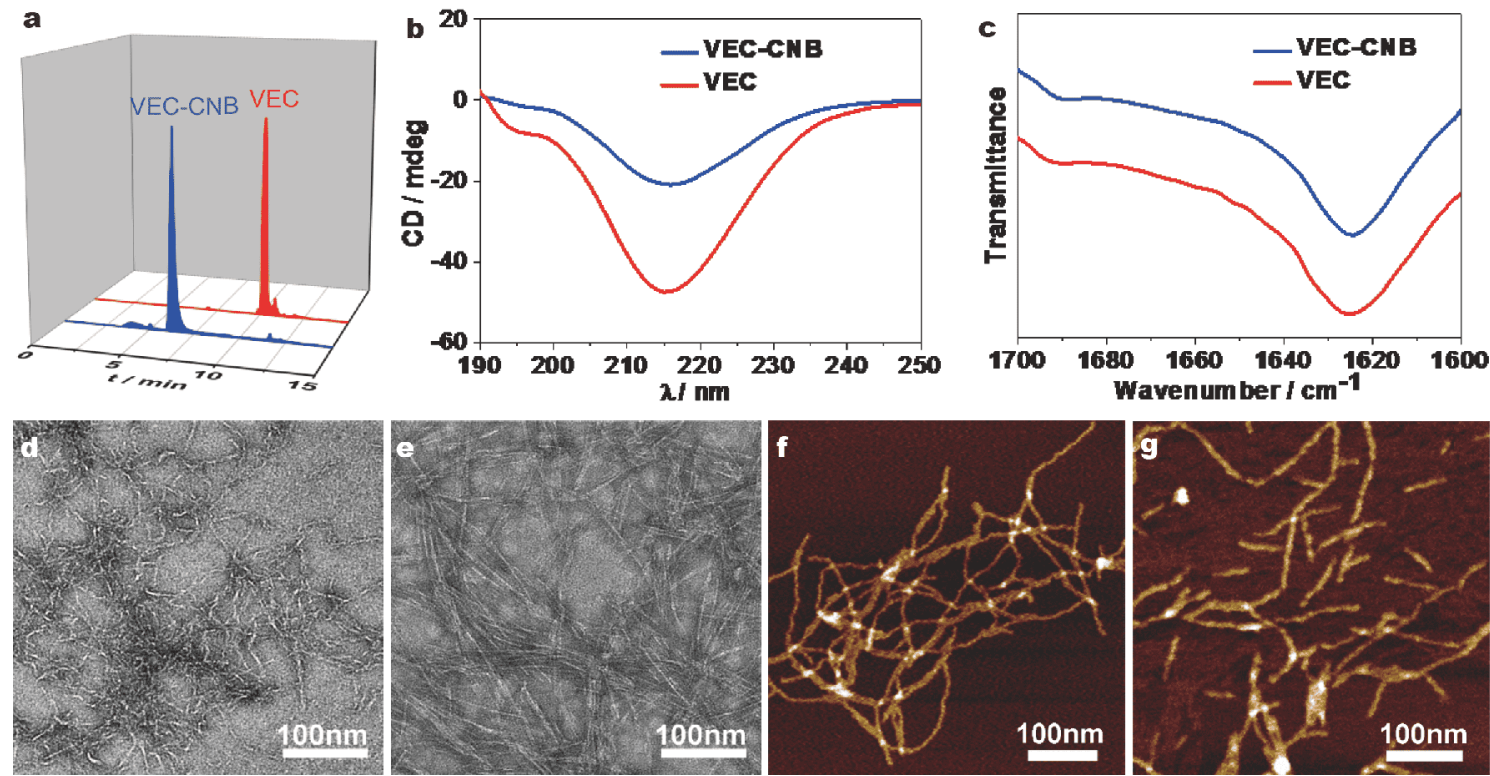

Figure 1 Self-assembly of peptides VEC-CNB and VEC in annealed solutions. (a) HPLC traces of peptide VEC-CNB and its photo-cleavage product peptide VEC. CD (b) and FTIR (c) spectra of peptides VEC-CNB and VEC. TEM and AFM images of the assemblies formed by peptide VEC-CNB (d, f) and VEC (e, g). TEM samples were stained by uranyl acetate (2\%). 


\section{Rheological experiments}

Rheology measurements were performed on a TA Instruments AR 1500ex rheometer operating in an oscillatory mode. The peptide samples were dropped onto a $40 \mathrm{~mm}$ parallel plate, and the plate was equipped with a solvent trap of silicon oil in order to prevent evaporation of the solvent. To measure the mechanical property of the static hydrogel VEC-CNB, the annealed solution of peptide VEC-CNB $\left(12 \mathrm{mmol} \mathrm{L}^{-1}, 375 \mu \mathrm{L}\right)$ was transferred to the plate $\left(400 \mu \mathrm{m}\right.$ gap), whereas $\mathrm{CaCl}_{2}$ solution $\left(2 \mathrm{~mol} \mathrm{~L}^{-1}, 25 \mu \mathrm{L}\right)$ was homogeneously loaded on the top cone. The mechanical property of the static hydrogel VEC was estimated by transferring the annealed intermediate hydrogel VEC $\left(12 \mathrm{mmol} \mathrm{L}^{-1}, 390 \mu \mathrm{L}\right)$ to the plate stage (400 $\mu \mathrm{m}$ gap) and homogeneously loading $\mathrm{CaCl}_{2}$ solution $\left(2 \mathrm{~mol} \mathrm{~L}^{-1}, 10 \mu \mathrm{L}\right)$ on the top cone. In addition, transferring the fresh intermediate hydrogel VEC $\left(12 \mathrm{mmol} \mathrm{L}^{-1}, 390 \mu \mathrm{L}\right)$ on the plate stage $(400 \mu \mathrm{m}$ gap) and loading $\mathrm{CaCl}_{2}$ solution on the top cone allowed for characterization of the mechanical properties of the mechanically dynamic hydrogels VEC-MD. Peptide hydrogels were immediately subjected to a 2 -h time sweep test. After reaching a well-defined hydrogelation equilibrium, a series of frequency sweep and strain amplitude sweep tests were subsequently carried out. Dynamic time sweep experiments were performed with a frequency and strain amplitude of $1 \mathrm{rad} \mathrm{s}^{-1}$ and $0.1 \%$, respectively. The dynamic frequency sweep tests were studied over a range of frequencies from 0.1 to $100 \mathrm{rad} \mathrm{s}^{-1}$ at $0.1 \%$ constant strain to measure the linear viscoelastic storage $\left(G^{\prime}\right)$ and loss modulus $\left(G^{\prime \prime}\right)$, respectively. The oscillatory strain amplitude sweep tests were investigated over a range of strain amplitude from $0.1 \%$ to $10 \%$ at a fixed frequency of $1 \mathrm{rad} \mathrm{s}^{-1}$.

\section{Hydrogel preparation and stem cell culture}

The lyophilized powder of peptide VEC-CNB was weighed and sterilized under autoclaving for $20 \mathrm{~min}$. The annealed solution of peptide VEC-CNB $\left(12 \mathrm{mmol} \mathrm{L}^{-1}\right)$ was prepared by dissolving the sterilized powder in ammonia water $(\mathrm{pH} \mathrm{10)}$ and adjusting the $\mathrm{pH}$ to 5.6 with aqueous $\mathrm{HCl}$. Thermodynamically static hydrogel VEC$\mathrm{CNB}$ was prepared by adding $80 \mu \mathrm{L}$ of annealed $12 \mathrm{mmol} \mathrm{L}^{-1}$ peptide VEC-CNB solution and $5 \mu \mathrm{L} \mathrm{CaCl}_{2}$ solution ( $2 \mathrm{~mol} \mathrm{~L}^{-1}, 4$ equiv.) to each well of 96-well plate. To the annealed solution of peptide VEC-CNB $\left(12 \mathrm{mmol} \mathrm{L}^{-1}, 80 \mu \mathrm{L}\right), 6$ equiv. of $\mathrm{CaCl}_{2}$ solution $\left(2 \mathrm{~mol} \mathrm{~L}^{-1}, 3 \mu \mathrm{L}\right)$ was added and exposed to UV light for $6 \mathrm{~h}$ to form the intermediate hydrogel VEC. Static hydrogel VEC was prepared by adding $80 \mu \mathrm{L}$ of annealed intermediate hydrogel VEC and $2 \mu \mathrm{L} \mathrm{CaCl}_{2}$ solutions $\left(2 \mathrm{~mol} \mathrm{~L}^{-1}\right)$ to each well of 96-well plate. The mechanically dynamic hydrogel VEC-MD was prepared by adding $80 \mu \mathrm{L}$ of fresh intermediate hydrogel VEC and $2 \mu \mathrm{L} \mathrm{CaCl}_{2}$ solution $\left(2 \mathrm{~mol} \mathrm{~L}^{-1}\right)$ to each well of 96 -well plate. After incubating at room temperature for $30 \mathrm{~min}$, the hydrogelcontaining 96-well plates were centrifuged at $2000 \mathrm{r} \mathrm{min}^{-1}$ for $10 \mathrm{~min}$ and washed with $100 \mu \mathrm{L}$ of Dulbecco's modified Eagle medium (DMEM) until the color of medium was retained. The growth medium used for culturing V6.5 mESCs consists of 85\% DMEM (high glucose DMEM, GIBCO), $15 \%$ fetal bovine serum (FBS, Hyclone), $2 \mathrm{mmol} \mathrm{L}^{-1}$ L-glutamine, $5000 \mathrm{U} \mathrm{mL}^{-1}$ penicillin and streptomycin, $0.1 \mathrm{mmol} \mathrm{L}^{-1}$ nonessential amino acids (Invitrogen), $0.1 \mathrm{mmol} \mathrm{L}^{-1}$ 2-mercaptoethanol (Sigma), and $1000 \mathrm{U} \mathrm{mL}^{-1}$ leukemia inhibitory factor (LIF, ESGRO, Chem-icon).

\section{YAP-GFP OE HeLa cell culture}

YAP (Yes-associated protein)-GFP overexpression (OE) HeLa cell line was cultured in growth medium consisting of $90 \%$ high glucose DMEM, $10 \%$ FBS, $2 \mathrm{mmol} \mathrm{L}^{-1} \mathrm{~L}-$ glutamine, $5000 \mathrm{U} \mathrm{mL}^{-1}$ penicillin and streptomycin. The YAP-GFP OE HeLa cells were cultured on the surface of peptide hydrogels or the plate directly for $48 \mathrm{~h}$. Hoechst 33342 (Sigma) was used for nuclei staining. Fluorescence imaging was carried out by using an Olympus IX81 microscope. The numbers of the cells with cytoplasmic, nuclear, and cyto-nuclear distribution of YAP were counted based on the fluorescence images.

\section{RESULTS AND DISCUSSION}

We studied the photo-cleavage of the $\mathrm{CNB}$ groups in peptide VEC-CNB using analytic RP-HPLC. Exposure of peptide VEC-CNB to UV light $(365 \mathrm{~nm})$ led to the shift of its retention at $6.3 \mathrm{~min}$ in the HPLC trace to $10.2 \mathrm{~min}$ for a product (Fig. 1a), which possesses a molecular mass corresponding to the non-caged peptide VEC (Figs S2S4). These results are indicative of the photo-induced quantitative conversion of peptide VEC-CNB to VEC upon UV light irradiation. We further characterized the secondary structures formed by peptide VEC-CNB and its photo-cleavage product peptide VEC by using $\mathrm{CD}$ spectroscopy and FTIR (Fig. 1b, c). CD spectrum of peptide VEC-CNB predominately displayed a minimal band at $216 \mathrm{~nm}$, suggesting formation of $\beta$-sheets by peptide VEC-CNB. CD spectrum of peptide VEC exhibited a minimal Cotton effect peak at $216 \mathrm{~nm}$ as well (Fig. 1b), implying maintenance of $\beta$-sheet conformation of peptide VEC $[40,41]$. We found that at an identical 
concentration, the intensity of the $\beta$-sheet $C D$ signal of peptide VEC is higher than that of peptide VEC-CNB, indicating increase of the $\beta$-sheet population formed by peptide VEC compared with peptide VEC-CNB $[40,41]$. This increase is attributed to the enhanced stability of the $\beta$-sheets formed by peptide VEC potentially resulting from the elongated length of the sequences within the cross- $\beta$ assemblies compared with peptide VEC-CNB. The secondary structures formed by peptides VEC-CNB and VEC were confirmed by FTIR studies (Fig. 1c). FTIR spectra of peptides VEC-CNB and VEC showed two transmittance bands at 1624 and $1692 \mathrm{~cm}^{-1}$ associated with the vibration absorption of amide I bonds, suggesting formation of anti-parallel $\beta$-sheets by both of them $[42,43]$. The combined CD and FTIR results support our hypothesis of formation of the VEC-CNB bilayer assemblies with two oppositely protruded segments along the nanofibers and the VEC bilayer assemblies with fulllength sequences (Scheme 1), and thereby leading to the probability of creation of the mechanically dynamic hydrogels undergoing the photoinduced directional domain sliding movement.

We investigated the morphology of the assemblies formed by peptides VEC-CNB and VEC by TEM and AFM (Fig. 1d-g). TEM images showed that peptides VEC-CNB and VEC assembled into flexible and rigid nanofibers with a width of 4.21 and $5.85 \mathrm{~nm}$, respectively (Fig. S7). The widths of the VEC-CNB and VEC nanofibers are consistent with the lengths of the hydrophobic interfaces between approximately 8- or 13-residue long segments, respectively. This consistency indicates the hydrophobic collapse occurs to peptides VEC-CNB and VEC involving partial- or full-length domain, further suggesting that the hydrophilic CNB-caged cysteine residue serves as the stopper for the hydrophobic interface between anti-parallel organized VEC-CNB monomers. The fully hydrophobic collapse of peptide VEC potentially leads to increase of the $\beta$-sheet population compared with peptide VEC-CNB as revealed by CD studies. We confirmed the morphology of the assemblies formed by peptides VEC-CNB and VEC by carrying out AFM experiments (Fig. 1f, g). AFM studies displayed flexible and rigid nanofibers formed by peptides VEC-CNB and VEC, respectively. The height of the resulting flexible or rigid nanofiber was determined to be 2.07 and $2.21 \mathrm{~nm}$, respectively (Fig. S8), which is corresponding to the widths of the bilayers of peptides VEC-CNB and VEC (Figs S9, S10). The combination of the conformational and morphological results allows us to elucidate the potential mechanism for the self-assembly of peptides VEC-
CNB and VEC (Fig. 2). Analogue to conventional alternating hydrophilic and hydrophobic peptides, both peptides VEC-CNB and VEC assemble into nanofibers driven by anti-parallel $\mathrm{H}$-bonding interactions and hydrophobic interactions at the valine-interfaces. While the partial hydrophobic collapse of the domains promotes the self-assembly of peptide VEC-CNB into flexible nanofibers appended with two protruded disordered segments, the stiff nanofibers are formed by VEC caused by the full hydrophobic collapse. This mechanism potentially allows for the directional domain sliding motion promoted by the hydrophobic collapse transition upon photo-cleavage of the CNB groups within peptide VEC-CNB bilayers.

We estimated the hydrogelation behavior of peptide VEC-CNB in dark or upon UV light irradiation by rheological experiments to prepare the thermodynamically static and mechanically dynamic hydrogels (Fig. 3 and Figs S11-S14). We found that adding of calcium chloride (10 equiv.) to the annealed solution of peptide VEC-CNB in dark led to immediate hydrogelation and formation of thermodynamically static hydrogel VEC-CNB with a storage modulus of $828.5 \mathrm{~Pa}$ (Fig. 3a, b). However, upon UV irradiation of the annealed solution of peptide VEC-CNB for $6 \mathrm{~h}$, CD spectrum of the resulting VEC solution showed that the intensity of $\beta$-sheet signals is close to that of the annealed solution of peptide VEC (Fig. S15). This indicates partial completion of the
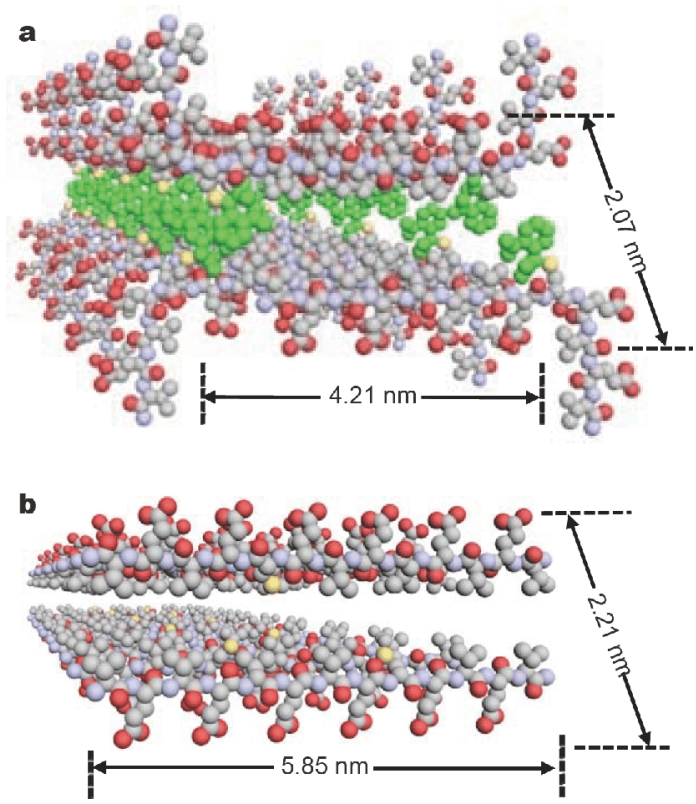

Figure 2 Graphic illustration of the self-assembly of peptides VEC-CNB (a) and VEC (b) via intermeshing two domains at their hydrophobic interface with two protruded segments or blunt termini, respectively. 


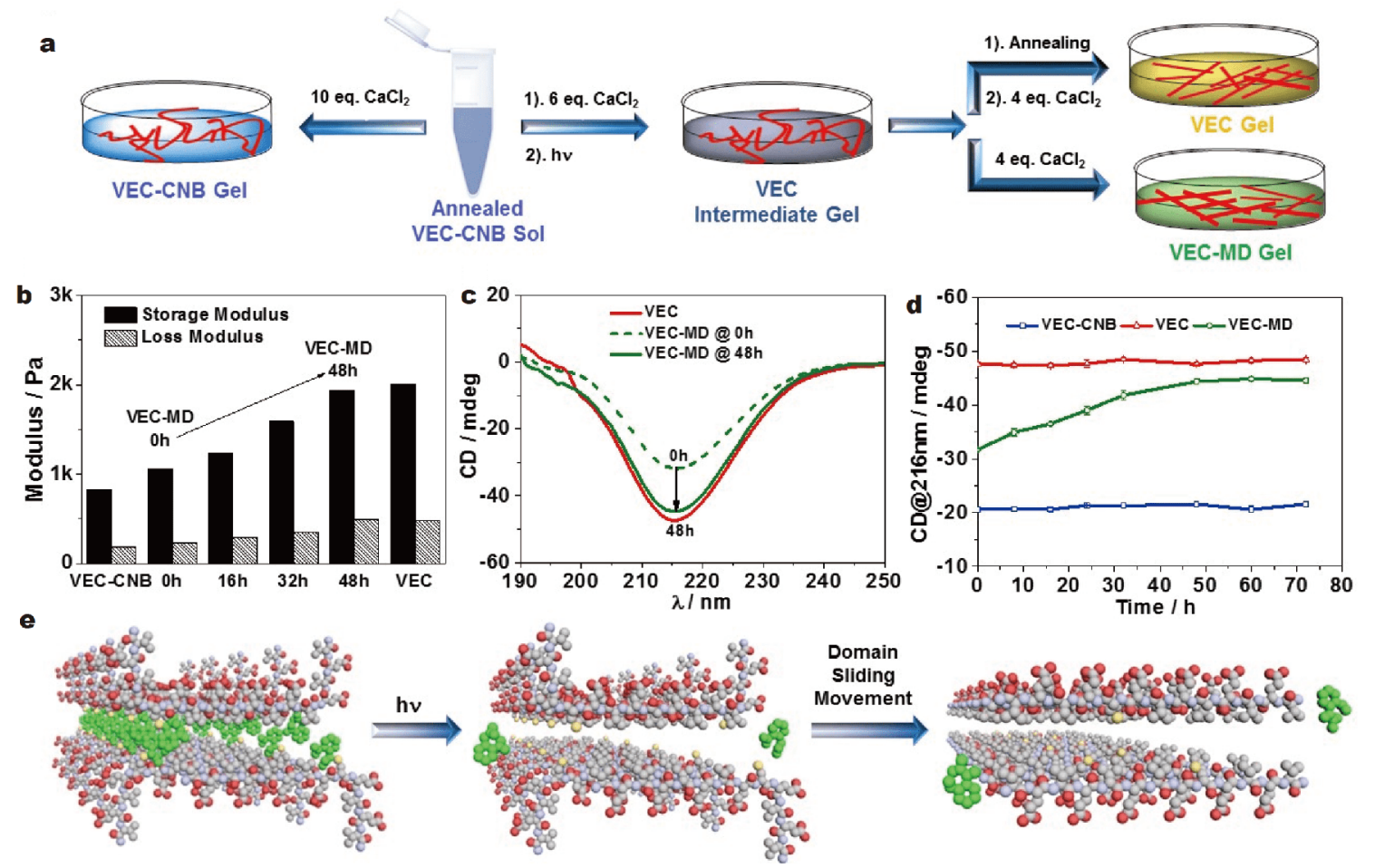

Figure 3 (a) Schematic illustration of preparation of mechanically dynamic hydrogel VEC-MD, static hydrogels VEC-CNB and VEC. (b) Timedependent mechanical properties of hydrogel VEC-MD compared with that of static hydrogels VEC-CNB and VEC. (c) CD spectra of hydrogels VEC and hydrogel VEC-MD at 0 and $48 \mathrm{~h}$. (d) Changes of CD signals (@216 nm) of static hydrogels VEC-CNB and VEC, and hydrogels VEC-MD as a function of time. (e) Schematic representation of the mechanically dynamic process within the MD hydrogels based on photo-cleavage of CNB groups and directional domain sliding motion.

transition from the partial to full hydrophobic collapse within the non-equilibrated VEC bilayers. To prepare mechanically dynamic peptide hydrogels undergoing directional mechanical movement, we reduced the rate of the photo-induced hydrophobic collapse transition via trapping the non-equilibrated VEC bilayers in intermediate hydrogels. Hence, irradiation of the annealed solution of VEC-CNB mixing with 6 equiv. of $\mathrm{CaCl}_{2}$ for $6 \mathrm{~h}$ led to preparation of the intermediate hydrogel VEC composed of VEC bilayers exhibiting a $\beta$-sheet signal intensity close to that of peptide VEC-CNB, indicating the non-equilibrated state for VEC bilayers within the intermediate hydrogel (Fig. 3c). Annealing the intermediate hydrogel VEC and adding additional 4 equiv. of $\mathrm{CaCl}_{2}$ resulted in formation of thermodynamically static hydrogel VEC with a storage modulus of $2007.0 \mathrm{~Pa}$ (Fig. 3a, b). Based on the intrinsic relationship between the length of alternating polar and nonpolar sequences and the stiffness of resulting hydrogels, increase of the stiffness of static hydrogel VEC compared with static hydrogel VEC-CNB suggests the partial or full hydrophobic collapse within the bilayers of VEC-CNB and
VEC, respectively. Meanwhile, adding 4 equiv. of $\mathrm{CaCl}_{2}$ to the intermediate hydrogel VEC led to formation of the mechanically dynamic hydrogel VEC-MD. It is worth noting that the amount of $\mathrm{CaCl}_{2}$ used for promoting hydrogelation is identical for all the three hydrogels to prevent the metal-coordination effect on the mechanical property of hydrogels. Time-dependent rheological studies showed that the mechanical properties of hydrogel VEC-MD exhibited a storage modulus $1060.3 \mathrm{~Pa}$ close to the value of the static hydrogel VEC-CNB (Fig. 3b), indicating an initial stage of hydrogel VEC-MD composed of non-equilibrated VEC bilayers stabilized by the partial hydrophobic collapse involving partial segments. After $48 \mathrm{~h}$, the storage modulus of hydrogel VEC-MD gradually increased to a value of $1936.5 \mathrm{~Pa}$ close to that of static hydrogel VEC, suggesting the occurrence of the hydrophobic interactions involving the full sequence (Fig. S16). The time-dependent rheological studies demonstrate that the mechanically dynamic hydrogel VEC-MD undergo the transition from a partial to full hydrophobic collapse within the non-equilibrated VEC bilayers.

We carried out the time-dependent CD experiments to 
gain insight into the underlying mechanical for the hydrophobic collapse transition of non-equilibrated VEC bilayers within the mechanically dynamic hydrogel VECMD. A significant increase of the $\beta$-sheet signal intensity of hydrogel VEC-MD over $48 \mathrm{~h}$ indicates the enhanced population of $\beta$-sheets (Fig. S17), indicative of the transition from partial to full hydrophobic collapse within the non-equilibrated VEC bilayers in hydrogel VEC-MD (Fig. 3d). In contrast, CD spectra of static hydrogels VECCNB and VEC showed the constant intensity of the $\beta$ sheet signal at $216 \mathrm{~nm}$ (Figs S18, S19), suggesting stable organization of the equilibrated peptide bilayers in the hydrogels VEC-CNB and VEC (Fig. 3d). Due to the intimate relationship between the organizing fashion and the $\beta$-sheet population, we attributed the gradual increase of the $\beta$-sheet $\mathrm{CD}$ signal intensity to the directional domain sliding movements within the non-equilibrated VEC bilayers, rather than the dynamic dissociationreassociation process during the hydrophobic collapse transition within hydrogel VEC-MD. The time-dependent $\mathrm{CD}$ studies demonstrate that photo-cleavage of the CNB groups within equilibrated VEC-CNB bilayers generates the non-equilibrated VEC bilayers that undergoing the thermodynamically favorable hydrophobic collapse transition through a directional domain sliding motion, thus leading to the mechanically dynamic hydrogels with intrinsic mechanical movements (Fig. 3e).

Creation of the mechanically dynamic hydrogels allows us to investigate the effect of the directional mechanical movement on the differentiation of murine V6.5 ESCs when utilizing the hydrogels as artificial ECM (Fig. 4). Under the self-renewing condition by adding LIF, ESCs were cultured on the surface of static hydrogels VEC$\mathrm{CNB}$ and VEC or mechanically dynamic hydrogel VECMD for $1 / 2 / 4$ days (Fig. 4b). ESCs on hydrogels proliferate slower than ESCs on gelatin. Regardless, after 4-day culturing, an obvious increase of cell number for ESCs on hydrogels indicates the comparable capability for ESC growth by hydrogel VEC-CNB, VEC, and VEC-MD. In addition, alkaline phosphatase (AP) staining of ESCs cultured on hydrogels and gelatin for 4 days showed the comparable number of colonies (Fig. 4c). These results suggest the excellent biocompatibility of the three hydrogels as artificial cellular matrices for ESCs.

We further investigated the differentiation of ESCs on static hydrogels VEC-CNB and VEC or mechanically dynamic hydrogel VEC-MD. Regardless of the culture surface, the expression levels of the pluripotency genes Sox2, Nanog, and Oct4 in ESCs in the absence of LIF are significant lower than those in ESCs in the presence of

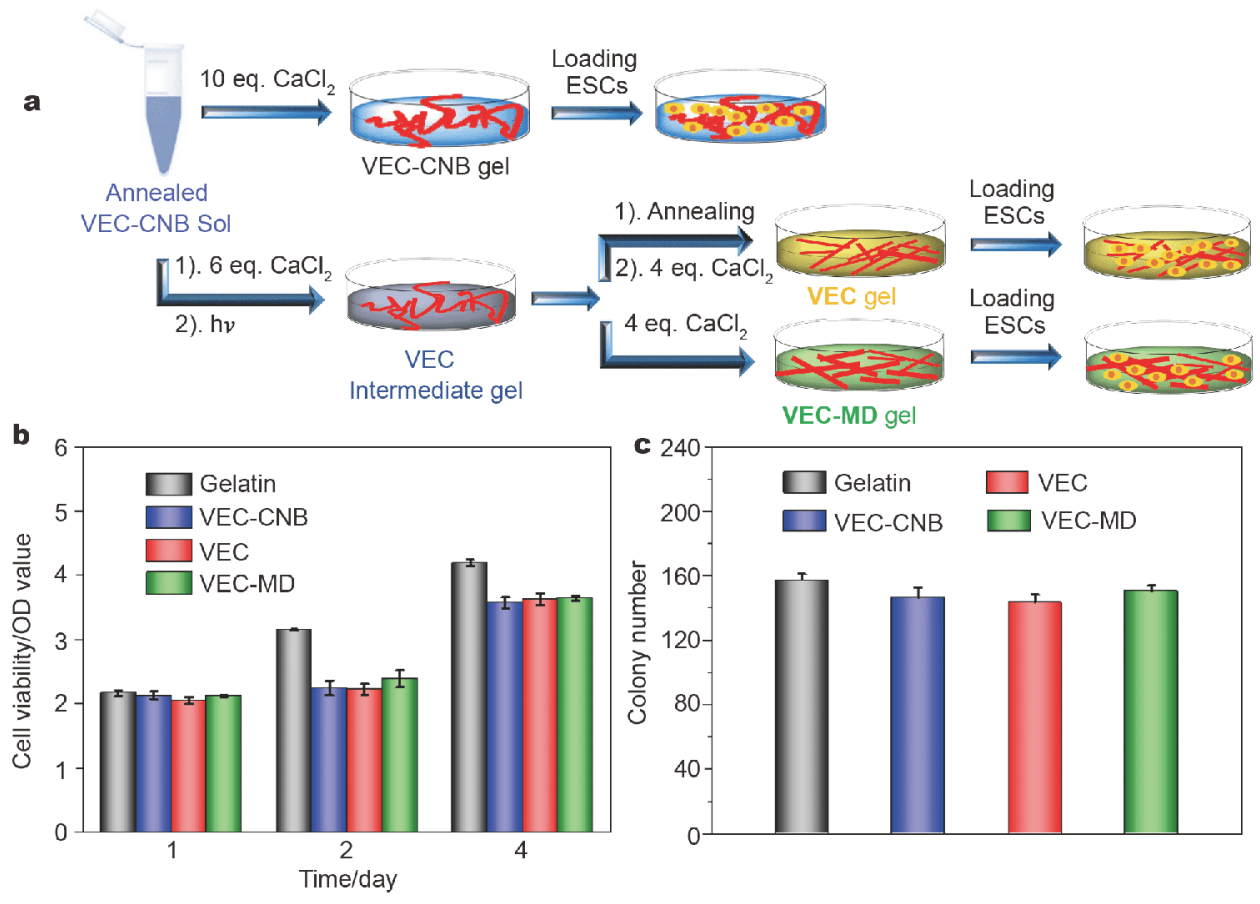

Figure 4 Differentiation of murine V6.5 ESCs on the surface of hydrogels. (a) Graphic illustration of culturing ESCs on static hydrogels VEC-CNB and VEC, and hydrogels VEC-MD. Cell viability detected by CCK-8 (b) and counted colony number (c) of ESCs cultured on gelatin, static hydrogels VEC-CNB, VEC, and hydrogel VEC-MD in serum/LIF medium. 
LIF (Fig. 5a), indicating the differentiation of ESCs upon LIF withdrawal when cultured on peptide hydrogels. In particular, all the pluripotency genes in ESCs cultured on hydrogel VEC-MD are expressed at the lowest expression level compared with ESCs on static hydrogels VEC-CNB and VEC and gelatin, implying the excellent capability of hydrogel VEC-MD for promoting the differentiation of ESCs. In addition, we analyzed the differentiation of ESCs by detecting the expression of the three germ layer markers, including paired box protein-6 (Pax6) and neuroepithelial stem cell protein (Nestin) as ectodermal markers, Fetal liver kinase-1 (Flk1) and Brachyury (T) as mesodermal markers, and Gata6 and Gata4 as endodermal markers, via quantitative reverse-transcription polymerase chain reaction (qRT-PCR) (Fig. 5b). The expression levels of the three germ layer markers in ESCs on hydrogels or gelatin without LIF significantly increased compared with ESCs cultured on gelatin in the presence of LIF, indicative of the efficient differentiation of ESCs when using hydrogels as matrices.

We found that ectodermal markers Pax6 and Nestin were expressed at higher levels in ESCs cultured on hydrogels than those in ESCs on gelatin, suggesting that hydrogels VEC-CNB, VEC, and VEC-MD promoted the biased differentiation of ESCs towards the ectodermal lineage. In particular, the levels of Pax6 and Nestin in ESCs on hydrogel VEC-MD were further enhanced compared with ESCs on hydrogels VEC-CNB and VEC,
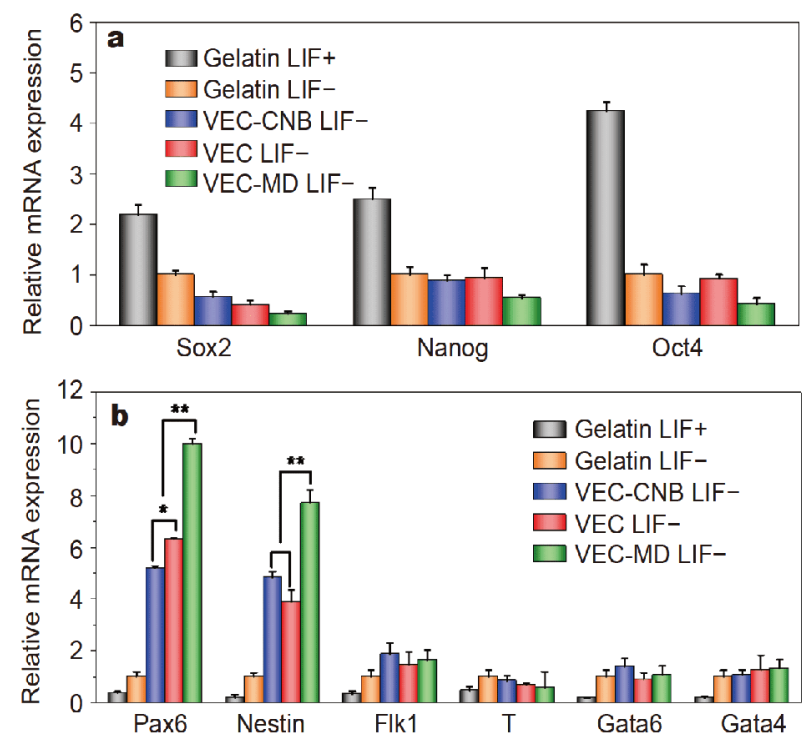

Figure 5 Differentiation of ESCs by LIF withdrawal on gelatin or hydrogels for 4 days by recording the pluripotency markers: Sox2, Nanog, and Oct4 (a), as well the germ layer markers: Pax6, Nestin, Flk1, Brachyury (T), Gata6, and Gata4 (b) via qRT-PCR analysis. whereas the levels of Pax6 and Nestin in ESCs on static hydrogels VEC-CNB and VEC were only slightly different. The slight difference in the expression levels of Pax6 and Nestin in ESCs on hydrogels VEC-CNB and VEC are potentially associated with the distinct mechanical properties of the hydrogels. Considering hydrogel VEC-MD exhibiting the stiffness gradient between those of hydrogels VEC-CNB and VEC and the identical chemical component between hydrogels VEC-MD and VEC, we attribute the enhanced level of Pax6 and Nestin in ESCs on hydrogel VEC-MD to the photo-induced directional domain sliding movement within hydrogel VEC-MD, rather than the mechanical property of the hydrogels. Based on the critical role of Pax6 and Nestin in physiological functions, the biased differentiation of ESCs into ectodermal lineages potentially facilitates the production of skin and nerve cells for skin transplantation and treatment of neurological diseases $[44,45]$. Therefore, the results demonstrate that the mechanically dynamic hydrogel VEC-MD possesses the great potency for cell replacement therapy and tissue regeneration by promoting the ectodermal differentiation of ESCs.

To test the possibility that the mechanical movement within the hydrogels promotes the biased ESC differentiation through a mechanotransduction pathway, we monitored the activity of the Hippo pathway in HeLa cells cultured on static hydrogels VEC-CNB and VEC or mechanically dynamic hydrogel VEC-MD, using a YAPGFP OE HeLa cell line (Fig. 6). The Hippo signaling pathway is a mechanotransduction pathway regulating cell proliferation, apoptosis, and self-renewal in response to mechanical cues [46]. The Hippo pathway consists of a kinase cascade, during which the downstream transcription co-activator YAP is phosphorylated. Previous studies revealed that mechanical cues, such as stiff ECM, promote nuclear translocation of unphosphorylated YAP for downstream gene activation [47]. Hence, monitoring the cyto-nuclear distribution of YAP allows for estimation of the mechanical cues perceived by cells. After 2-day culturing of YAP-GFP OE HeLa on hydrogels VEC-CNB, VEC, or VEC-MD (Fig. S21), fluorescence images showed that culturing the HeLa cells on hydrogel VEC-MD led to a higher percentage of cells with nuclear localized YAP (Fig. 6a and Fig. S22), compared with the cells on the hydrogels VEC-CNB and VEC and on culture plate (Fig. 6b). The difference of the distribution of YAP in cells cultured on hydrogels VEC-CNB and VEC might be attributed to the distinct hydrogel stiffness. However, the improved translocation of YAP into nucleus in cells cultured on hydrogel VEC-MD is likely associated with 


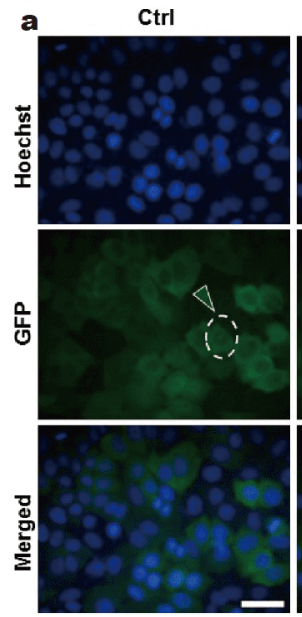

VEC-CNB

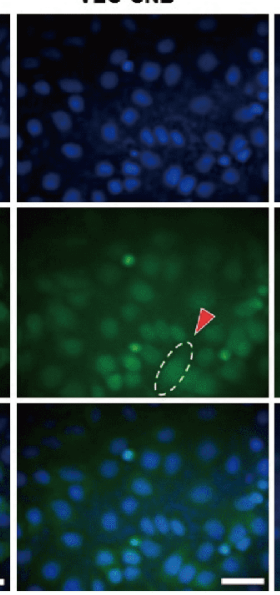

VEC

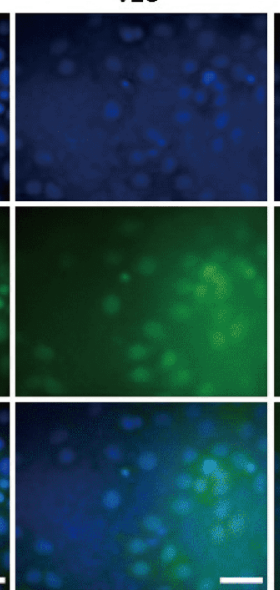

VEC-MD

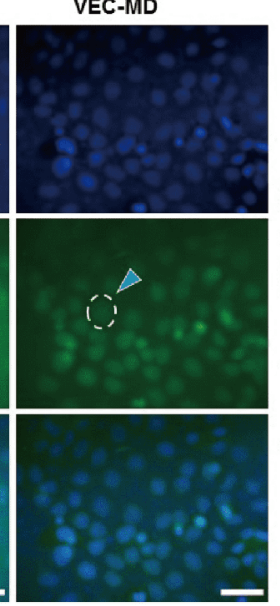

b

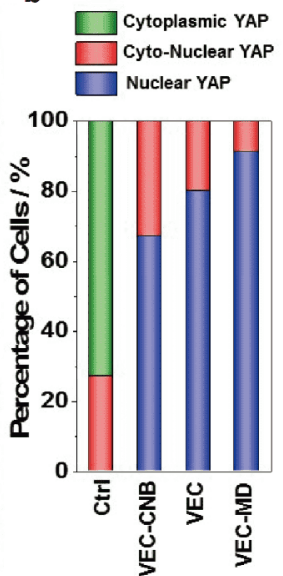

Figure 6 Localization of YAP in YAP-GFP OE HeLa cells cultured on hydrogels. (a) Fluorescence images of YAP-GFP OE HeLa cells cultured on plate surface, static hydrogels VEC-CNB, VEC, and MD hydrogel VEC-MD for 2 days. Green, blue, and red triangles mark cells in dash ellipses with cytoplasmic, nuclear, or cyto-nuclear distribution of YAP protein, respectively. Scale bars are $50 \mu \mathrm{m}$. (b) Cell counts for cytoplasmic, nuclear, or cytonuclear distribution of YAP protein.

the directional domain sliding movement within hydrogel VEC-MD, due to the intermediate stiffness of hydrogel VEC-MD compared to hydrogels VEC-CNB and VEC. The YAP-GFP localization assays demonstrate that cells can sense the mechanical cues generated by the mechanical movements within the mechanically dynamic hydrogel VEC-MD. Therefore, it also supports a potential mechanotransduction mechanism for the biased ectodermal differentiation of ESCs guided by the photoinduced directional mechanical movement within the hydrogels.

\section{CONCLUSIONS}

In summary, we have designed and synthesized an alternating hydrophilic and hydrophobic peptide with a photocaged cysteine residue and created mechanically dynamic hydrogels undergoing photo-induced directional domain sliding movement. While the photocaged peptide assembled into flexible nanofibers induced by the hydrophobic interactions involving only partial-length sequence due to the hydrophilicity of the caged cysteine residues, the non-caged peptide formed rigid nanofibers caused by the hydrophobic collapse involving the fulllength sequence due to the hydrophobicity of non-caged cysteine residues. Therefore, upon UV light irradiation, the assemblies of the caged peptide were converted into non-equilibrated non-caged peptide bilayers that underwent the directional domain sliding motion induced by the hydrophobic collapse transition. Hydrogelation of the non-equilibrated non-caged peptide bilayers by adding calcium chloride resulted in formation of hydrogels undergoing directional domain sliding movement, which guided the biased ectodermal differentiation of murine ESCs. YAP-GFP localization assays revealed that the translocation of a mechanotransduction protein YAP into nucleus was facilitated by the mechanically dynamic hydrogels, demonstrating generation of mechanical cues by the mechanical movement when utilizing the hydrogels as ECM and thereby indicating a potential mechanotransduction mechanism for the biased ectodermal differentiation of ESCs. Our results of the mechanically dynamic hydrogels promoting ectodermal differentiation of ESCs, which can be further differentiated into skin and nerve cells and applied for skin transplantation and treatment of neurological diseases, demonstrate that the mechanically dynamic hydrogels are promising biomaterials for regenerative medicine. Alternative to dynamic hydrogels, the non-equilibrated peptide assemblies potentially serve as one new type of platforms to associate with cells in solution and affect their cellular behavior including proliferation, movement, and cargo uptake due to the intrinsic mechanical signals within peptide assemblies, thus facilitating development of functional biomaterials towards high performance in disease therapy.

Received 15 August 2019; accepted 11 September 2019; published online 29 October 2019

1 Engler AJ, Sen S, Sweeney HL, et al. Matrix elasticity directs stem cell lineage specification. Cell, 2006, 126: 677-689

2 Lecuit T, Lenne PF. Cell surface mechanics and the control of cell 
shape, tissue patterns and morphogenesis. Nat Rev Mol Cell Biol, 2007, 8: 633-644

3 Friedland JC, Lee MH, Boettiger D. Mechanically activated integrin switch controls a5 $\beta 1$ function. Science, 2009, 323: 642-644

4 Vining KH, Mooney DJ. Mechanical forces direct stem cell behaviour in development and regeneration. Nat Rev Mol Cell Biol, 2017, 18: 728-742

5 Bao M, Xie J, Huck WTS. Recent advances in engineering the stem cell microniche in 3D. Adv Sci, 2018, 5: 1800448

6 Dalby MJ, Gadegaard N, Tare R, et al. The control of human mesenchymal cell differentiation using nanoscale symmetry and disorder. Nat Mater, 2007, 6: 997-1003

7 McMurray RJ, Gadegaard N, Tsimbouri PM, et al. Nanoscale surfaces for the long-term maintenance of mesenchymal stem cell phenotype and multipotency. Nat Mater, 2011, 10: 637-644

8 Ondeck MG, Kumar A, Placone JK, et al. Dynamically stiffened matrix promotes malignant transformation of mammary epithelial cells via collective mechanical signaling. Proc Natl Acad Sci USA, 2019, 116: 3502-3507

9 Huang G, Li F, Zhao X, et al. Functional and biomimetic materials for engineering of the three-dimensional cell microenvironment. Chem Rev, 2017, 117: 12764-12850

10 Brown TE, Anseth KS. Spatiotemporal hydrogel biomaterials for regenerative medicine. Chem Soc Rev, 2017, 46: 6532-6552

11 Guvendiren M, Burdick JA. Stiffening hydrogels to probe shortand long-term cellular responses to dynamic mechanics. Nat Commun, 2012, 3: 792

12 Hadden WJ, Young JL, Holle AW, et al. Stem cell migration and mechanotransduction on linear stiffness gradient hydrogels. Proc Natl Acad Sci USA, 2017, 114: 5647-5652

13 Zhang J, Cheng C, Cuellar-Camacho JL, et al. Thermally responsive microfibers mediated stem cell fate via reversibly dynamic mechanical stimulation. Adv Funct Mater, 2018, 28: 1804773

14 Yang C, Tibbitt MW, Basta L, et al. Mechanical memory and dosing influence stem cell fate. Nat Mater, 2014, 13: 645-652

15 Wen JH, Vincent LG, Fuhrmann A, et al. Interplay of matrix stiffness and protein tethering in stem cell differentiation. Nat Mater, 2014, 13: 979-987

16 Chaudhuri O, Gu L, Klumpers D, et al. Hydrogels with tunable stress relaxation regulate stem cell fate and activity. Nat Mater, 2015, 15: 326-334

17 Ma Y, Lin M, Huang G, et al. 3D spatiotemporal mechanical microenvironment: a hydrogel-based platform for guiding stem cell fate. Adv Mater, 2018, 30: 1705911

18 Hörner M, Raute K, Hummel B, et al. Phytochrome-based extracellular matrix with reversibly tunable mechanical properties. Adv Mater, 2019, 31: 1806727

19 Zhang S, Gelain F, Zhao X. Designer self-assembling peptide nanofiber scaffolds for 3D tissue cell cultures. Semin Cancer Biol, 2005, 15: 413-420

20 Wang H, Yang Z, Adams DJ. Controlling peptidebased hydrogelation. Mater Today, 2012, 15: 500-507

21 Du X, Zhou J, Shi J, et al. Supramolecular hydrogelators and hydrogels: from soft matter to molecular biomaterials. Chem Rev, 2015, 115: 13165-13307

22 Silva GA, Czeisler C, Niece KL, et al. Selective differentiation of neural progenitor cells by high-epitope density nanofibers. Science, 2004, 303: 1352-1355

23 Gelain F, Silva D, Caprini A, et al. BMHP1-derived self-assembling peptides: hierarchically assembled structures with self-healing propensity and potential for tissue engineering applications. ACS Nano, 2011, 5: 1845-1859

24 Ye K, Wang X, Cao L, et al. Matrix stiffness and nanoscale spatial organization of cell-adhesive ligands direct stem cell fate. Nano Lett, 2015, 15: 4720-4729

25 Ferris DP, Zhao YL, Khashab NM, et al. Light-operated mechanized nanoparticles. J Am Chem Soc, 2009, 131: 1686-1688

26 SzymaŃski W, Yilmaz D, Koçer AĞ, et al. Bright ion channels and lipid bilayers. Acc Chem Res, 2013, 46: 2910-2923

27 Peng $\mathrm{H}$, Li XF, Zhang $\mathrm{H}$, et al. A microRNA-initiated DNAzyme motor operating in living cells. Nat Commun, 2017, 8: 14378

28 García-López V, Chen F, Nilewski LG, et al. Molecular machines open cell membranes. Nature, 2017, 548: 567-572

29 Liu D, García-López V, Gunasekera RS, et al. Near-infrared light activates molecular nanomachines to drill into and kill cells. ACS Nano, 2019, 13: 6813-6823

30 Lussis P, Svaldo-Lanero T, Bertocco A, et al. A single synthetic small molecule that generates force against a load. Nat Nanotech, 2011, 6: 553-557

31 Juluri BK, Kumar AS, Liu Y, et al. A mechanical actuator driven electrochemically by artificial molecular muscles. ACS Nano, 2009, 3: $291-300$

32 Berná J, Leigh DA, Lubomska M, et al. Macroscopic transport by synthetic molecular machines. Nat Mater, 2005, 4: 704-710

33 Lou S, Wang X, Yu Z, et al. Peptide tectonics: encoded structural complementarity dictates programmable self-assembly. Adv Sci, 2019, 335: 1802043

34 Bowerman CJ, Liyanage W, Federation AJ, et al. Tuning $\beta$-sheet peptide self-assembly and hydrogelation behavior by modification of sequence hydrophobicity and aromaticity. Biomacromolecules, 2011, 12: 2735-2745

35 Haines LA, Rajagopal K, Ozbas B, et al. Light-activated hydrogel formation via the triggered folding and self-assembly of a designed peptide. J Am Chem Soc, 2005, 127: 17025-17029

36 Geisler IM, Schneider JP. Evolution-based design of an injectable hydrogel. Adv Funct Mater, 2012, 22: 529-537

37 Muraoka T, Cui H, Stupp SI. Quadruple helix formation of a photoresponsive peptide amphiphile and its light-triggered dissociation into single fibers. J Am Chem Soc, 2008, 130: 2946-2947

38 He M, Li J, Tan S, et al. Photodegradable supramolecular hydrogels with fluorescence turn-on reporter for photomodulation of cellular microenvironments. J Am Chem Soc, 2013, 135: 18718-18721

39 Smith DJ, Brat GA, Medina SH, et al. A multiphase transitioning peptide hydrogel for suturing ultrasmall vessels. Nat Nanotech, 2016, 11: 95-102

40 Fasman GD. Circular Dichroism and the Conformational Analysis of Biomolecules. New York: Plenum Publishers, 1996

41 Yu Z, Tantakitti F, Palmer LC, et al. Asymmetric peptide nanoribbons. Nano Lett, 2016, 16: 6967-6974

42 Haris PI, Chapman D. The conformational analysis of peptides using fourier transform IR spectroscopy. Biopolymers, 1995, 37: 251-263

43 Yu Z, Tantakitti F, Yu T, et al. Simultaneous covalent and noncovalent hybrid polymerizations. Science, 2016, 351: 497-502

44 Suter DM, Tirefort D, Julien S, et al. A Sox1 to Pax6 switch drives neuroectoderm to radial glia progression during differentiation of mouse embryonic stem cells. Stem Cells, 2009, 27: 49-58

45 Bernal A, Arranz L. Nestin-expressing progenitor cells: function, identity and therapeutic implications. Cell Mol Life Sci, 2018, 75: $2177-2195$ 
46

Yu FX, Zhao B, Panupinthu N, et al. Regulation of the Hippo-YAP pathway by G-protein-coupled receptor signaling. Cell, 2012, 150: 780-791

47 Dupont S, Morsut L, Aragona M, et al. Role of YAP/TAZ in mechanotransduction. Nature, 2011, 474: 179-183

Acknowledgements This work was supported by the National Key R\&D Program of China (2018YFC1313003), the Fundamental Research Funds for the Central Universities, the National Natural Science Foundation of China (21774065 and 31622038), and the Natural Science Foundation of Tianjin (18JCQNJC14100 and 18JCJQJC48400).

Author contributions Cheng $\mathrm{Z}$ and $\mathrm{Yu} \mathrm{Z}$ conceived and designed the study. Cheng $Z$ and Song $S$ carried out the preparation and characterization of the hydrogels. Nai S and Chen L designed and performed all the cellular studies. Cheng $\mathrm{Z}$ prepared the manuscript with assistance from Nai S. Chen $\mathrm{L}$ and $\mathrm{Yu} \mathrm{Z}$ revised the manuscript. All the authors discussed the results and have the approval of the submission of the final version of the manuscript.

Conflict of interest The authors declare that they have no conflict of interest.

Supplementary information online version of the paper.

Supporting data are available in the

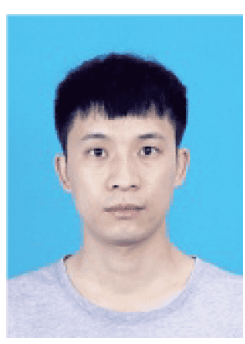

Zhifei Cheng received his MSc degree from Hefei University of Technology in 2017. Then he continued his study as a $\mathrm{PhD}$ candidate under the guidance of Prof. Zhilin Yu at Nankai University. His current research interest focuses on the development of peptide-based dynamic materials for biomedical applications.

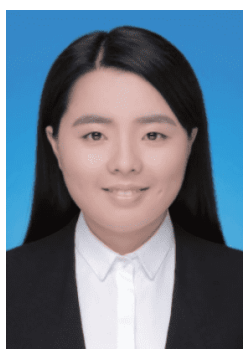

Shanshan Nai received her MSc degree from the College of Life Sciences, Capital Normal University in 2018. She is now a PhD student in Prof. Lingyi Chen's laboratory at the College of Life Sciences, Nankai University. Her current interest focuses on novel Mek1 downstream targets in maintaining the pluripotency of embryonic stem cells.

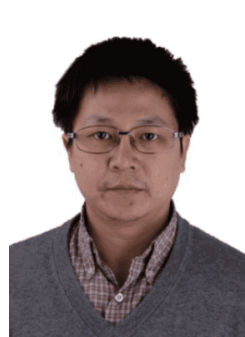

Lingyi Chen is a professor and vice dean of the College of Life Sciences, Nankai University, and an awardee of the National Science Fund for Excellent Young Scholars. He obtained his PhD degree in biochemistry from Northwestern University in 2004, and then conducted his postdoctoral research in Harvard University. $\mathrm{He}$ established his laboratory at Nankai University in 2008, and his research interests include molecular mechanism of pluripotency maintenance in embryonic stem cells and molecular regulation of early mouse embryonic development.

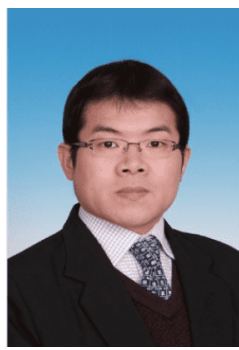

Zhilin Yu was awarded his $\mathrm{PhD}$ degree under the supervision of Prof. Stefan Hecht at the Humboldt-Universität zu Berlin in 2013. He conducted his postdoctoral training with Prof. Samuel I. Stupp at Northwestern University focusing on self-assembly of peptide-based amphiphilic molecules. In 2017, he started his independent career at the Institute of Polymer Science of Nankai University. His current research interests focus on the self-assembly of peptides into dynamic nanostructures and their broad applications as biomaterials including disease diagnosis and therapy.

\section{含光诱导片段定向滑动多肽水凝胶诱导胚胎干细 胞的外胚层分化}

\author{
程志非 ${ }^{1 \dagger}$, 能姗姆 ${ }^{2 \dagger}$, 宋树金金, 陈凌懿 ${ }^{2 *}$, 余志林 $^{1 *}$
}

摘要 由细胞内行为或外源力引起的干细胞拿中存在的机械信号 对干细胞的自我恢复和分化等基本功能具有重要影响. 然而, 关于 具有分子机械运动产生的内在机械信号的人工细胞外基质鲜有报 道. 在此, 我们报道了含光诱导片段定向滑动的机械动态水凝胶的 合成及其作为人工细胞外基质在调节胚胎干细胞(ESC)分化中的 功能. 通过引入光笼蔽的半胱氨酸残基调控亲疏水交替多肽的自 组装制备机械动态水凝胶. 光笼蔽多肽组装体在光照射下转化为 热力学非平衡的非笼蔽多肽双分子层, 其进一步发生热力学有利 的疏水性塌陷转变诱导的片段定向滑动. 在机械动态水凝胶上培 养鼠胚胎干细胞, 该片段定向滑动诱导干细胞向外胚层谱系定向 分化. 进一步揭示了机械动态水凝胶促进机械转导蛋白 $\mathrm{YAP}$ 进入 细胞核, 表明其用于ESCs定向分化的潜在机械转导机制. 细胞定向 分化结果表明了机械动态水凝胶作为潜在的生物材料, 有望用于 疾病治疗和组织再生. 